\title{
A Critical Analysis of the Mean-Field Approximation for the Calculation of the Magnetic Moment in the Friedel-Anderson Impurity Model
}

\author{
Gerd Bergmann \\ Department of Physics \\ University of Southern California \\ Los Angeles, California 90089-0484 \\ e-mail: bergmann@usc.edu \\ D: $\backslash 0$ aa $\backslash$ Aa_tex $\backslash$ A_paper $\backslash$ Arb_- $\backslash B 152$
}

July 6, 2018

\begin{abstract}
It is shown that the calculation of the magnetic moment of a Friedel-Anderson impurity in mean-field theory is unreliable. A class of approximate solutions, which contains the mean-field solution as an element, is expressed in rotated Hilbert space and optimized. The optimal state has considerably lower energy than the mean field solution and requires almost twice the Coulomb exchange $U$ to become magnetic. Since most moment calculations of magnetic impurities, for example the spin-density-functional theory, use the mean-field approximation the resulting magnetic moments have to be critically reexamened.
\end{abstract}

PACS: 75.20.Hr,

The properties of magnetic impurities in a metal is one of the most intensively studied problems in solid state physics. Although some of the experimental anomalies were already discovered in the 1930's, it is still a subject 
of great interest. The work of Friedel [1] and Anderson [2] laid the foundation to understand why some transition metal impurities form a magnetic local moment, while others don't. They considered a host with an s-band in which a transition metal atom is dissolved. The s-electrons can hop onto the d-impurity via the hopping matrix element $V_{s d}$. The ten-fold degeneracy of a real d-impurity is simplified and reduced to a two-fold degeneracy for spin up and spin down. If both states are occupied they repel each other due to the Coulomb exchange energy. This yields the Friedel-Anderson Hamiltonian

$$
H_{F A}=\sum_{\sigma}\left\{\sum_{\nu=1}^{N} \varepsilon_{\nu} c_{\nu \sigma}^{*} c_{\nu \sigma}+E_{d} d_{\sigma}^{*} d_{\sigma}+\sum_{\nu=1}^{N} V_{s d}(\nu)\left[d_{\sigma}^{*} c_{\nu \sigma}+c_{\nu \sigma}^{*} d_{\sigma}\right]\right\}+U n_{d+} n_{d-}
$$

Here a finite s-band with $N$ states is used. The $c_{\nu \sigma}^{*}$ and the $d_{\sigma}^{*}$ are the creation operators of the (free) s-electrons and the d-impurity. The $d_{\sigma}^{*}$-states are assumed to be orthogonal to the s-states $c_{\nu}^{*}$ (in the following I denote single electron states by their creation operator).

In the limit of $V_{s d}=0$ and $E_{d}<\varepsilon_{F}, E_{d}+U>\epsilon_{F}$ the d-impurity is magnetic. Anderson concluded that the magnetic moment survives for small but finite $V_{s d}$ and derived the criteria for a magnetic state and the size of the moment in a mean-field approximation. He found a magnetic state if the product of $U g_{d}>1$ where $g_{d}$ is the additional density of states of the d-resonance.

Kondo [3] brought a new twist into the magnetic impurity problem when he showed that multiple scattering of conduction electrons by a magnetic impurity yields a divergent contribution to the resistance in perturbation theory. In the following three decades a large number of sophisticated methods were applied to better understand and solve the Kondo and Friedel-Anderson model, and it was shown that at zero temperature a Friedel-Anderson impurity is in a non-magnetic singlet state. However, above the Kondo temperature the impurity shows a magnetic moment, and there is a great interest in the size of this moment.

There is a large body of research in which the magnetic moment of impurities is calculated [4, [5], 6], 7], 8]. Generally spin-density-functional theory is used for this task. Within this theory the electronic structure of the host and the impurity is calculated from first principles without any adjustable parameters. In particular the strength of the Coulomb and exchange interaction are obtained from first principles. However, in the final step the mean-field method is applied to obtain the local magnetic moment. 
Although this is a zero-temperature calculation (where the impurity should be in the Kondo singlet state) it is generally argued that such a calculation yields the magnetic moment above the Kondo temperature (which, at lower temperatures, is hidden in the singlet state).

In this letter I will show that the mean-field result for the magnetic moment of impurities is not reliable. By rewriting the mean-field solution in a rotated basis and optimizing the solution I obtain solutions which are much lower in energy, require a much larger critical $U$ for the formation of a moment and yield smaller moments. And this despite the fact that the improved solution has the same structure (in the rotated basis) as the mean-field solution. Since there is a large body of spin-density-functional theory calculations for magnetic impurities, a reevaluation of this method might be required.

I start with Anderson's (potentially) magnetic state which he obtained as a mean-field solution. Anderson replaced the Hamiltonian $H_{F A}$ by

$$
\begin{aligned}
& H_{m f}=H_{F+}+H_{F-}-U\left\langle n_{d+}\right\rangle\left\langle n_{d-}\right\rangle \\
& H_{F \sigma}=\Sigma_{\nu} \varepsilon_{\nu} c_{\nu \sigma}^{*} c_{\nu \sigma}+\sum_{\nu=1}^{N} V_{s d}(\nu)\left[d_{\sigma}^{*} c_{\nu \sigma}+c_{\nu \sigma}^{*} d_{\sigma}\right]+E_{d, \sigma} d_{\sigma}^{*} d_{\sigma}
\end{aligned}
$$

where $\left\langle n_{d+}\right\rangle$ and $\left\langle n_{d_{-}}\right\rangle$are the average occupation numbers of the states $d_{+}^{*}$ and $d_{-}^{*}$ and $E_{d, \sigma}=\left(E_{d}+U\left\langle n_{d,-\sigma}\right\rangle\right)$. The solution of the mean-field method requires the diagonalization of two Friedel resonance Hamiltonians $H_{F \sigma}$ with self consistent values for $\left\langle n_{d+}\right\rangle$ and $\left\langle n_{d-}\right\rangle$. This straight-forward numerical calculation yields the mean-field ground-state energy $E_{m f}$ and the magnetic moment $\mu_{m f}$. The energy of the bare magnetic state $E_{b . m}$. is subtracted from $E_{m f}$ where

$$
E_{b . m .}=2 \sum_{\nu=1}^{n} \varepsilon_{\nu}+E_{d}-\varepsilon_{n}
$$

is the ground-state energy for $V_{s d}=0$ and $E_{d}<\varepsilon_{F}, E_{d}+U>\varepsilon_{F}$.

For the numerical calculation an s-band with a constant density of states is used, ranging from -1 to +1 . This band is divided into $N=48$ equal cell. Each s-sub-band is half filled, i.e. the number of occupied states in each spin sub-band is $n=N / 2$.In Fig. 1 the numerical results for $E_{m f}-E_{b . m}$. are plotted for $\left|V_{s d}\right|^{2}=0.05$. The Coulomb repulsion $U$ is varied between 0.2 and 1.2. Together with the Coulomb repulsion the $d^{*}$-state energy $E_{d}$ is 
varied so that $E_{d}$ and $\left(E_{d}+U\right)$ lie symmetrically about the Fermi energy, i.e. $E_{d}=-\frac{1}{2} U$.

In the mean-field calculation the impurity is non-magnetic for $U<U_{c r} \approx$ 0.275 . For $U>U_{c r}$ the spin up and down sub-bands split. The resulting magnetic moments are plotted in Fig.2 (curve with circles).

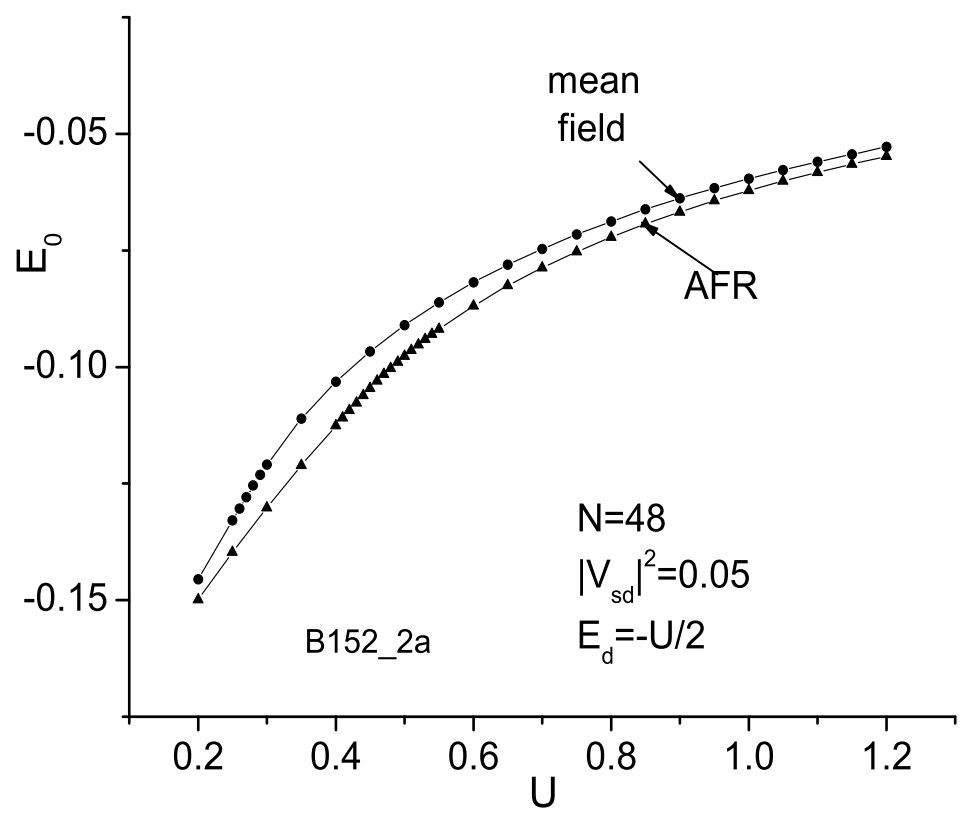

Fig.1: A comparison between the ground-state energies of the mean-field calculation and the AFR method (see text below).

Since the mean-field solution is the product of two $n$-electron states of the two Friedel Hamiltonians $F_{F+}$ and $H_{F-}$ we consider these solutions of the Friedel Hamiltonian (31) in some detail. As shown in ref. [9, 10] the exact ground state of $H_{F}$ with $n$ (spinless) electrons can be written in the form

$$
\Psi_{F}=\left[A^{\prime} a_{0}^{*}+B^{\prime} d^{*}\right] \prod_{i=1}^{n-1} a_{i}^{*} \Phi_{0}
$$


Here $\Phi_{0}$ is the vacuum state and $a_{0}^{*}$ is a sister state to $d^{*}$ which is built from the states of the s-band

$$
a_{0}^{*}=\sum_{\nu=1}^{N} \alpha_{\nu}^{0} c_{\nu}^{*}
$$

Ref. [10] shows how to calculate the coefficients $\alpha_{\nu}^{0}$ from the parameters of the Hamiltonian $H_{F}$ and the occupation number $n$. The $a_{i}^{*}$ are orthogonal to $a_{0}^{*}$ and to each other and their $(N-1)$ sub-matrix of the s-band Hamiltonian $H_{0}=\sum \varepsilon_{\nu} n_{\nu}$ is diagonal (see equ. 8 ). The states $a_{i}^{*}$ are uniquely determined from the state $a_{0}^{*}$. Their form is

$$
a_{i}^{*}=\sum_{\nu=1}^{N} \alpha_{\nu}^{i} c_{\nu}^{*}
$$

The $a_{i}^{*}(1 \leq i \leq N-1)$ together with $a_{0}^{*}$ represent a new basis.

In this new basis the free electron Hamiltonian $H_{0}=\sum_{\nu=1}^{N} \varepsilon_{\nu} c_{\nu}^{*} c_{\nu}$ takes the form

$$
H_{0}=\sum_{i=1}^{N-1} E(i) a_{i}^{*} a_{i}+E(0) a_{0}^{*} a_{0}+\sum_{i=1}^{N-1} V_{f r}^{a}(i)\left[a_{0}^{*} a_{i}+a_{i}^{*} a_{0}\right]
$$

In the Hamiltonian (8) the $a_{0}^{*}$-state represents an artificial resonance state. I will call it in honor of Friedel an Artificial Friedel Resonance state (AFR state). It is a sister state to the state $d^{*}$.

The full (spin independent) Friedel Hamiltonian can be written as

$$
\begin{aligned}
H_{F} & =\sum_{i=1}^{N-1} E(i) a_{i}^{*} a_{i}+E(0) a_{0}^{*} a_{0}+E_{d} d^{*} d+V_{s d}^{a}(0)\left[d^{*} a_{0}+a_{0}^{*} d\right] \\
& +\sum_{i=1}^{N-1} V_{s d}^{a}(i)\left[d^{*} a_{i}+a_{i}^{*} d\right]+\sum_{i=1}^{N-1} V_{f r}^{a}(i)\left[a_{0}^{*} a_{i}+a_{i}^{*} a_{0}\right]
\end{aligned}
$$

where 


$$
\begin{aligned}
E(i) & =\sum_{\nu} \alpha_{\nu}^{i} \varepsilon_{\nu} \alpha_{\nu}^{i} \\
E(0) & =\sum_{\nu} \alpha_{\nu}^{0} \varepsilon_{\nu} \alpha_{\nu}^{0} \\
V_{s d}^{a}(i) & =\sum_{\nu} V_{s d}(\nu) \alpha_{\nu}^{i} \\
V_{f r}^{a}(i) & =\sum_{\nu} \alpha_{\nu}^{i} \varepsilon_{\nu} \alpha_{\nu}^{0}
\end{aligned}
$$

In the Hamiltonian (9) the $d^{*}$-state and the localized $a_{0}^{*}$-state are on equal footing. The second line in equ. (91) yields the hopping between $a_{i}^{*}$ and $d^{*}$ (first term) and $a_{i}^{*}$ and $a_{0}^{*}$ (second term). For the state $\left(A^{\prime} a_{0}^{*}+B^{\prime} d^{*}\right)$ the individual hopping matrix elements cancel each other, making $\Psi_{F r}$ the ground state.

In the next step the mean-field solution is rewritten in the AFR-form of the Friedel ground state. Since the Hamiltonian consists of a Friedel Hamiltonian for each spin the mean-field state is the product of two states of the form of equ.(5). Therefore this mean-field state (the exact solution of the mean-field Hamiltonian) can be written as

$$
\begin{aligned}
\Psi_{0} & =\left[A_{-} a_{0-}^{*}+B_{-} d_{-}^{*}\right]\left[A_{+} a_{0+}^{*}+B_{+} d_{+}^{*}\right] \prod_{\sigma, i=1}^{n-1} a_{i \sigma}^{*} \Phi_{0} \\
& =\left[A a_{0-}^{*} a_{0+}^{*}+B d_{-}^{*} a_{0+}^{*}+C a_{0-}^{*} d_{+}^{*}+D d_{-}^{*} d_{+}^{*}\right] \prod_{\sigma, i=1}^{n-1} a_{i \sigma}^{*} \Phi_{0} \\
& =A \Psi_{A}+B \Psi_{B}+C \Psi_{C}+D \Psi_{D}
\end{aligned}
$$

where

$$
\begin{array}{cccccc}
A_{+}^{2}+B_{+}^{2} & = & 1 & A_{-}^{2}+B_{-}^{2} & = & 1 \\
A & = & A_{+} A_{-}, & B & = & A_{+} B_{-} \\
C & = & A_{-} B_{+}, & D & = & B_{+} B_{-}
\end{array}
$$

Each of the four states $\Psi_{A}, \Psi_{B}, \Psi_{C}$ and $\Psi_{D}$ is normalized, and they are all orthogonal to each other. In the magnetic solution one has $A_{+} \neq A_{-}$and 
$B_{+} \neq B_{-}$. Also the two rotated bases $\left\{a_{0+}^{*}, a_{i+}^{*}\right\}$ and $\left\{a_{0-}^{*}, a_{i-}^{*}\right\}$ are different in the magnetic state.

So far the many electron state in equ. (51) is the mean-field solution. This state consists of an electron background $\prod_{\sigma, i=1}^{n-1} a_{i \sigma}^{*} \Phi_{0}$ multiplied with the sum of four two-electron states, consisting of the combinations $\left[a_{0-}^{*} a_{0+}^{*}, d_{-}^{*} a_{0+}^{*}, a_{0-}^{*} d_{+}^{*}, d_{-}^{*} d_{+}^{*}\right]$ which have $S_{z}=0$. The mean-field wave function opens a new playing field for variation to find the optimal state. One can optimize the coefficients $A$, $B, C$ and $D$ while dropping the individual normalization conditions (12) and replacing them by

$$
A^{2}+B^{2}+C^{2}+D^{2}=1
$$

Far more important one can optimize the states $a_{0+}$ and $a_{0-}$.

For this purpose the Hamiltonian $H_{F A}$ is expressed in the bases $\left\{a_{0+}^{*}, a_{i+}^{*}\right\}$ and $\left\{a_{0-}^{*}, a_{i-}^{*}\right\}$. One obtains for the expectation value of the ground-state energy $E_{0}$

$$
\begin{aligned}
E_{0} & =A^{2}\left[E_{-}(0)+E_{+}(0)\right]+B^{2}\left[E_{-}(0)+E_{d}\right]+C^{2}\left[E_{+}(0)+E_{d}\right]+D^{2}\left[2 E_{d}+U\right] \\
& +2(A B+C D) V_{s d}^{-}(0)+2(A C+B D) V_{s d}^{+}(0)+\sum_{\sigma, i=1}^{n-1} E_{\sigma}(i)
\end{aligned}
$$

For a given set of states $\left\{a_{0 \pm}^{*}, a_{i \pm}^{*}\right\}$ the energy $E_{0}$ in eq. (14) depends on the coefficients $A, B, C$ and $D$. One obtains the lowest energy by varying $E_{0}$ with respect to these coefficients. This yields a $4 \times 4$ matrix for the coefficients vector $(A, B, C, D)$. The lowest eigenvalue gives the energy expectation value, and its eigenvector gives the coefficients. The resulting state I denote as the magnetic state $\Psi_{A F R}$ and the solution as the AFR solution.

The central part of the numerical calculation is the variation of the states $a_{0+}^{*}$ and $a_{0-}^{*}$ until the absolute minium of the energy is reached.

As in the mean-field theory the numerical calculation itself determines whether the lowest state possesses a magnetic moment or not. If the solution is magnetic then $a_{0+}^{*}$ and $a_{0-}^{*}$ approach different states and the coefficients $\mathrm{B}$ and $\mathrm{C}$ have different values. The resulting magnetic moment is defined as the difference in the occupation of the $d_{+}^{*}$ and $d_{-}^{*}$ states, i.e. $\mu=B^{2}-C^{2}$.

In Fig. 1 the energy expectation value $E_{0}$ of the optimal magnetic state $\Psi_{A F R}$ is plotted as the curve with the triangles (again the same energy $E_{b . m}$. 
has been subtracted). The new ground-state energy lies considerably below the mean-field energy.

In Fig. 2 the resulting magnetic moments that one obtains with the meanfield approximation and with the new method are plotted. One recognizes that the new solution suppresses the magnetic moment up to a considerably larger value of $U_{c r} \approx 0.46$. This is almost twice the value of the mean-field theory.

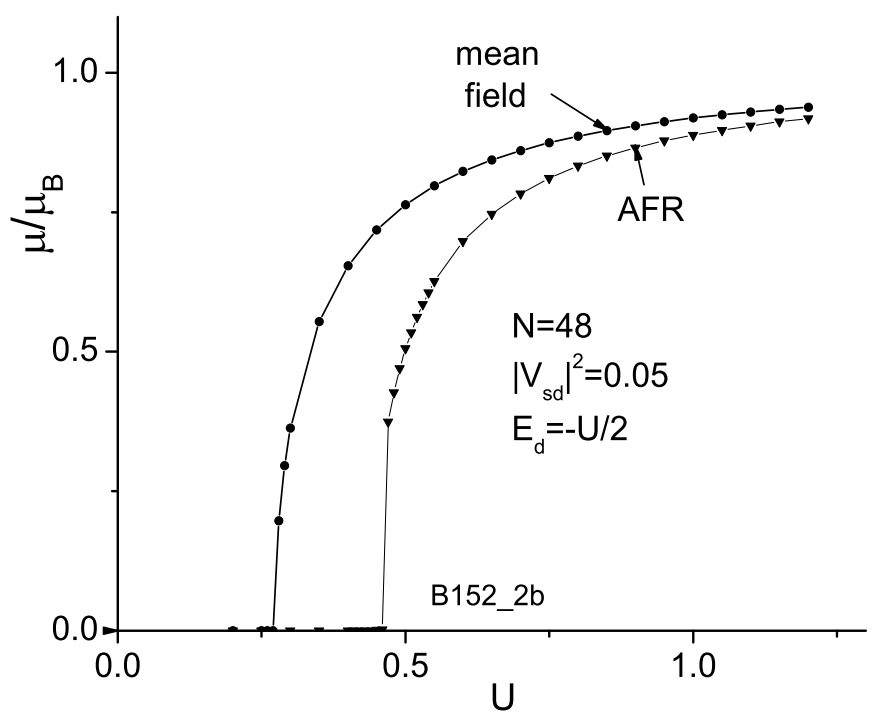

Fig.2: The magnetic moment as a function of the Coulomb energy $U$, using the mean-field solution and the AFR-method of the present calculation.

How do we have to interpret the fact that the AFR solution suppresses the magnetic moment up to a much larger critical Coulomb exchange interaction? Since this state has a lower energy expectation value, does this mean that its magnetic moment is more reliable? The author prefers a somewhat different interpretation. The Friedel-Anderson impurity does not like a broken symmetry. The mean-field approach does not give the multi-electron state any wiggling room. Only the values for $E_{d, \sigma}$ can be altered with increasing $U$. The AFR solution on the other hand possesses a lot more flexibility since 
the AFR states can adjust. Therefore the symmetric multi-electron state survives to a considerably larger Coulomb exchange interaction. It might be that neither state yields the right magnetic moment for the impurity. The present calculation raises serious questions about the mean-field approach. This might also apply to the spin-density-functional theory (SDFT) for magnetic impurities. This theory is a very complex theory and it is difficult to judge from the outside all the intricacies. It should yield the correct charge and spin densities for the correct functional. But in the final step the majority of SDFT calculations use a two-spin-fluid model where each electron fluid adjusts in the (mean) field of the other.

To conclude, in this paper an approximate solution for the Friedel-Anderson impurity is expressed in a rotated Hilbert space $\left\{a_{0 \pm}^{*}, a_{i \pm}^{*}\right\}$. Its center piece are two artificial resonance states $a_{0+}^{*}, a_{0-}^{*}$ for the spin up and down selectrons. They determine uniquely the remaining bases $\left\{a_{i+}^{*}\right\},\left\{a_{i-}^{*}\right\}$. The AFR states are combined with the d-electrons for spin up and down $d_{+}^{*}, d_{-}^{*}$ into two-electron states of total $S_{z}=0$, i.e. $\left[A a_{0-}^{*} a_{0+}^{*}+B d_{-}^{*} a_{0+}^{*}+C a_{0-}^{*} d_{+}^{*}+D d_{-}^{*} d_{+}^{*}\right]$. Then the $(n-1)$ lowest states of the two $(N-1)$ bases $\left\{a_{i \pm}^{*}\right\}$ are occupied yielding the s-electron background $\prod_{i=1, \sigma}^{n-1} a_{i \sigma}^{*} \Phi_{0}$. The compositions of the AFR states $a_{0+}^{*}, a_{0-}^{*}$ are calculated by numerical variation which rotates the selectron bases in Hilbert space.

The energy of the resulting state lies clearly below the mean-field solution. The critical value of the Coulomb exchange energy $U_{c r}$ for the formation of a magnetic moment is almost twice as large as in the mean-field solution. Since in many calculations of the magnetic moment of impurities the mean-field approximation is used one has to reevaluate the resulting moments. This may also apply to the impurity calculations which use the spin-density-functional theory because in the majority of these calculations the mean-field theory is used in the final analysis.

Since the ground state of the Friedel-Anderson impurity is a singlet state one might suspect that the structure of the new solution with the lower energy and smaller magnetic moment is somewhat closer to the singlet state than the mean-field solution. This is not the case. Both the mean-field and the present solution are in a symmetric state for small $U$; both show a similar asymmetry between spin up and down in the magnetic state. The mean-field solution belongs to the same class of wave functions as the here presented one (which are given by the general form of equ. (11)). 


\section{References}

[1] J.Friedel, Philos.Mag. 43, 153 (1952); Adv.Phys. 3, 446 (1954); Philos.Mag.Suppl. 7, 446 (1954); Can.J.Phys. 34, 1190 (1956); Nuovo Cimento Suppl. 7, 287 (1958); J. Phys.Radium 19, 38 (1958)

[2] P.W.Anderson, Phys.Rev. 124, 41 (1961)

[3] J.Kondo, Prog.Theor.Phys. 32, 37 (1964)

[4] S.K.Kwon and B.I.Min, Phys.Rev.Lett. 84, 3970 (2000)

[5] R.B.Sahu and L.Kleinman, Phys.Rev. B67, 094424 (2003)

[6] M.E.McHenry, J.M.MacLaren, D.D.VVendensky, M.E.Eberhart and M.L.Prueitt, Phys.Rev. B40, 10111 (1989)

[7] R.Podloucky, R.Zeller and P.H.Dederichs, Phys.Rev. B22, 5777 (1980)

[8] V.I.Anisimov and P.H.Dederichs, Solid State Commun., 84, 241 (1992)

[9] G.Bergmann, Z.Physik B102, 381 (1997)

[10] G.Bergmann, Eur.Phys.J.B2, 233 (1998) 